Revue internationale de l'économie sociale

Recma

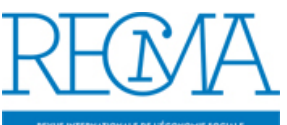

\title{
Les organisations coopératives et mutualistes face au marché et à la déréglementation
}

Jean-François Draperi

Numéro 278, octobre 2000

URI : https://id.erudit.org/iderudit/1023845ar

DOI : https://doi.org/10.7202/1023845ar

Aller au sommaire du numéro

Éditeur(s)

Institut de l'économie sociale (IES)

ISSN

1626-1682 (imprimé)

2261-2599 (numérique)

Découvrir la revue

Citer ce document

Draperi, J.-F. (2000). Les organisations coopératives et mutualistes face au marché et à la déréglementation. Revue internationale de l'économie sociale, (278), 4-4. https://doi.org/10.7202/1023845ar d'utilisation que vous pouvez consulter en ligne. 


\section{LES ORGANISATIONS COOPÉRATIVES ET MUTUALISTES FACE AU MARCHÉ ET À LA DÉRÉGLEMENTATION}

A près deux numéros thématiques consacrés à "Un siècle d'économie sociale " (avril 2000) et à « La coopération de crédit " (juillet 2000), ce numéro se présente sous une forme plus traditionnelle, autour de sujets variés. Il comprend deux importantes contributions au dernier colloque de l'Association pour le développement de la documentation sur l'économie sociale (Addes). Michel Forestier et René Mauget analysent le groupe coopératif agro-alimentaire. Cette contribution, dont nous publions la première partie - la seconde paraitra dans le prochain numéro -, porte sur le rapport entre cette évolution économique et organisationnelle et les valeurs et principes coopératifs: peut-on parler de dénaturation de l'entité coopérative, à l'instar de Vienney, qui analysait la possibilité d'une " mutation " lorsque la coopérative "n'est plus en mesure de conjuguer efficacité économique et cohésion sociale " (Vienney, L'économie sociale, 1994, p. 116)? L'analyse de Forestier et Mauget est sans doute la première en France à se pencher de façon aussi précise sur les modalités économiques et organisationnelles de la transformation de la coopérative en groupe complexe.

Henri Faivre étudie les incidences de la législation européenne sur les mutuelles françaises à partir de l'exemple des mutuelles de Vendée. L'auteur analyse l'évolution économique, et spécifiquement "lirruption du marché ", puis le débat contradictoire entre la FNMF, l'Etat français et la Commission des Communautés européennes. Il mesure ensuite l'impact des décisions européennes sur les mutuelles de Vendée. Enfin, l'auteur souligne le caractère international du problème: au-delà de la mutualité, c'est un statut européen des sociétés de personnes qui est en jeu.

Nous publions ensuite deux articles produits à partir de thèses de droit, primées par le Groupement national de la coopération (GNC). S'adossant aux travaux de C. Vienney, Marc Hérail souligne la place centrale du concept de double qualité dans la construction juridique. Son travail permet de légitimer la réglementation coopérative à partir $\mathrm{du}$ droit plutôt que sur la seule base des principes. Etudiant la même question, Patricia Papon-Vidal se penche sur la dissociation des qualités d'associé et de coopérateur. On notera l'intérêt particulier de cette question au moment où des formes innovantes d'économie sociale ne s'appuient pas nécessairement sur l'organisation coopérative classique spécifiée par la double qualité (voir par exemple Recma, $\mathrm{n}^{\circ}$ 275-276, pp. 124-135 et 168-182).

Ingo Bode étudie le devenir de la solidarité en France et en Allemagne à partir de l'évolution de l'assurance maladie. Après avoir souligné le caractère central de la solidarité dans l'économie sociale, l'auteur montre, au terme d'une analyse originale, les effets contrastés de la mise en concurrence des mutuelles. Certes, celles-ci ont parfois tenté de mettre en place des stratégies commerciales semblant tourner le dos à la solidarité, mais simultanément elles ont organisé, en France comme en Allemagne, la riposte à la dérive assurancielle. Ces luttes montrent que l'on peut sortir de la crise autrement que par la privatisation des systèmes de santé, à condition de se battre et d'innover pour faire face aux nouveaux défis liés à la pression économique. En fin de compte, conclut l'auteur, la déréglementation "pourrait leur rendre le sens social indispensable à leur pérennité » et représente ainsi " une nouvelle chance de survivre ". Enfin, Zvi Galor retrace la grande aventure des coopératives d'épargne et de crédit d'Israël, de leur création au début du XX⿳亠口冋口灬 siècle jusqu'à nos jours. S'appuyant parfois sur des sources inédites (comme un entretien avec Abraham Brener, ancien directeur de l'importante coopérative de crédit et d'épargne de Tel-Aviv), Zvi Galor montre l'importance de ce mouvement. S'attachant à détailler les règles de l'organisation de l'épargne et du crédit, il met en évidence la place de ces coopératives dans le développement, avant qu'elles ne se fédèrent et, finalement, se transforment en banques coopératives.

Jean-François Draperi 